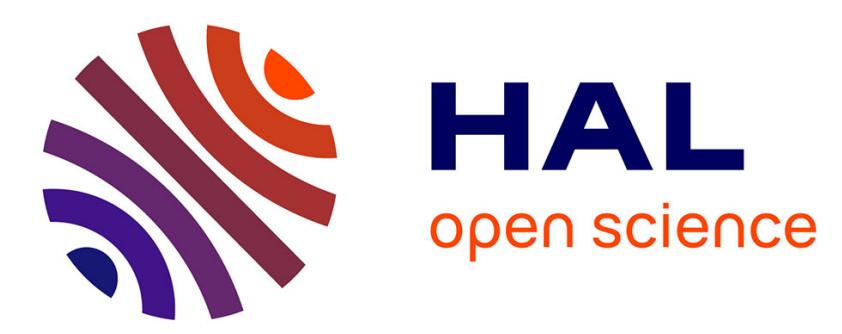

\title{
Selective Electrochemical Bleaching of the Outer Leaflet of Fluorescently Labeled Giant Liposomes
}

\author{
Ana Isabel Perez Jimenez, Lylian Challier, Eric Aït-Yahiatène, Jérôme \\ Delacotte, Eric Labbé, Olivier Buriez
}

\section{- To cite this version:}

Ana Isabel Perez Jimenez, Lylian Challier, Eric Aït-Yahiatène, Jérôme Delacotte, Eric Labbé, et al.. Selective Electrochemical Bleaching of the Outer Leaflet of Fluorescently Labeled Giant Liposomes. Chemistry - A European Journal, 2017, 10.1002/chem.201605786 . hal-01472848

\section{HAL Id: hal-01472848 \\ https: / hal.sorbonne-universite.fr/hal-01472848}

Submitted on 21 Feb 2017

HAL is a multi-disciplinary open access archive for the deposit and dissemination of scientific research documents, whether they are published or not. The documents may come from teaching and research institutions in France or abroad, or from public or private research centers.
L'archive ouverte pluridisciplinaire $\mathbf{H A L}$, est destinée au dépôt et à la diffusion de documents scientifiques de niveau recherche, publiés ou non, émanant des établissements d'enseignement et de recherche français ou étrangers, des laboratoires publics ou privés. 


\title{
Selective Electrochemical Bleaching of the Outer Leaflet of Fluorescently Labelled Giant Liposomes
}

\author{
Ana Isabel Perez Jimenez, ${ }^{[a]}$ Lylian Challier, ${ }^{[a]}$ Eric Aït-Yahiatène, ${ }^{[a]}$ Jérôme Delacotte, ${ }^{[a]}$ Eric Labbé, ${ }^{[a]}$ \\ and Olivier Buriez ${ }^{*[a]}$
}

\begin{abstract}
Electrochemistry and confocal fluorescence microscopy were successfully combined to selectively bleach and monitor the fluorescence of NBD-labelled phospholipids of giant liposomes. Three types of giant unilamellar vesicles have been investigated, the fluorescent phospholipids being localized either mainly on their outer-, inner-, or both inner/outer leaflets. We established that only the fluorescent lipids incorporated in the outer leaflet of the vesicles underwent electrochemical bleaching upon reduction. The relative fluorescence intensity decay was quantified all along the electrochemical extinction through an original Fluorescence Loss In Electrobleaching (FLIE) assay. As expected, the re-organization of the fluorescent phospholipids followed diffusion-driven dynamics. This was also evidenced by comparison with Fluorescence Loss In Photobleaching (FLIP) and the corresponding numerical model. The value of the lateral diffusion coefficient of phospholipids was found to be similar to that obtained by other methods reported in literature. This versatile and selective bleaching procedure appears reliable to explore important biological and pharmacological issues.
\end{abstract}

\section{Introduction}

Combination of electrochemistry with confocal or wide field fluorescence microscopy techniques is of high interest in the fields of analytical chemistry, biophysics and chemical physics. On the one hand, fluorescence microscopy allows imaging and fluorescence quantification. On the other hand, electrochemistry can be used to switch fluorescence on and off. ${ }^{[1]}$

The coupling of fluorescence microscopy with electrochemistry has notably proved to be a powerful approach in view of obtaining both time- and spatially-resolved information at the molecular level. Besides, several works reported in this field focus on the electrochemical establishment of $\mathrm{pH}$ or concentration gradients. In the first case, the main strategy relies on the use of $\mathrm{pH}$-sensitive fluorescent probes. This approach was well developed in the 1990s by Engstrom and coworkers to monitor, with a wide field fluorescence microscope, reactions producing or consuming protons at the level of an electrode. ${ }^{[2-4]}$ Following the work reported on the use of confocal Raman

[a] Dr. A.I. Perez Jimenez, Dr. L. Challier, E. Aït-Yahiatène, Dr. J. Delacotte, Prof. E. Labbé, Dr. O. Buriez

Ecole normale supérieure, PSL Research University, UPMC Univ

Paris 06, CNRS, Département de Chimie, PASTEUR, 24, rue

Lhomond, 75005 Paris, France

E-mail: Olivier.buriez@ens.fr

Supporting information for this article is given via a link at the end of the document.((Please delete this text if not appropriate)) microscopy to map molecular diffusion at microelectrodes, ${ }^{[5,6]}$ Unwin and colleagues successfully imaged in 2002, for the first time, three-dimensional $\mathrm{pH}$ gradients at electrode surfaces thanks to the use of a confocal laser scanning microscope which improves the axial and lateral resolutions. ${ }^{[7]}$ In this case, the local $\mathrm{pH}$ changes due to the electrochemical reduction of benzoquinone to hydroquinone were evidenced thanks to the presence of fluorescein which displayed $\mathrm{pH}$-dependent fluorescent signal. Confocal $\mathrm{pH}$ measurements in solution were also performed later by Boldt et al. in the presence of a scanning electrochemical microscope. ${ }^{[8]}$

Contrary to $\mathrm{pH}$-gradients, the establishment of concentrationgradients is mainly based on the use of molecules possessing both fluorescent and redox properties. In this case, the measured fluorescence is directly dependent on the potential applied. For instance, the diffusion concentration profile resulting from the oxidation of the fluorescent tris(bipyridine) ruthenium(II) into the non-fluorescent tris(bipyridine)-ruthenium(III) could be mapped by confocal microscopy. ${ }^{[9]}$ Very recently, Doneux and coworkers successfully coupled electrochemistry with confocal microscopy to investigate the electrochemical reactivity of the resazurin-resorufin fluorogenic couple and to reconstruct in $3 \mathrm{D}$ the diffusion layer of the fluorescent probe. ${ }^{[10]}$ Though decoupled from the electrochemical reaction of interest, we can also cite the work reported by Zhang and colleagues who developed an imaging tool called "fluorescence-enabled electrochemical microscopy", the principle of which being to associate a redox reaction to a fluorogenic reporter reaction through a closed bipolar electrode. ${ }^{[11-13]}$ Combination of fluorescence microscopy with electrochemistry can be also interesting in the presence of adsorbed fluorescent probes. For instance, Bizzotto et al. have used in situ fluorescence to investigate the reductive desorption of self-assembled monolayers made of fluorescently tagged alkylthiols. ${ }^{[14,15]}$ The technique relies on the fact that light emission from a fluorophore may be quenched when placed in close proximity $(\sim 50 \mathrm{~nm})$ to a bulk metallic surface. A fluorescent signal is therefore observed only when the adsorbed molecule becomes desorbed and separated from the electrode surface. More recently, the same group combined electrochemical measurements with in situ fluorescence imaging to monitor the poration / absorption / incorporation process of liposomes (100 nm in diameter), made of DOPC phospholipids, into a fluorescent adsorbed monolayer of octadecanol-covered surface. ${ }^{[16]}$

Fluorescent redox probes can be also trapped in biomolecular architectures such as liposomes which are used as biomembrane and protocell models. ${ }^{[17,18]}$ On the other hand, liposomes with diameters of a few micrometers and above are particularly attractive systems due to their accessibility to optical microscopy and various micromanipulation techniques. ${ }^{[19,20]}$ In 
this context, Bard and Zhan combined scanning electrochemical microscopy and fluorescence to probe the outside and the inside of individual giant liposomes $(15-50 \mu \mathrm{m}$ in diameter) in which the fluorescent tris(bipyridine)ruthenium(II) was encapsulated. ${ }^{[21]}$

However, our literature survey did not highlight any works involving redox fluorescent probes bound to phospholipids diffusing in lipid membranes. Several confocal microscopybased methods have been developed to switch off fluorescence locally in order to obtain physiological and physico-chemical information about cellular dynamics. ${ }^{[22]}$ Among them, Fluorescence Recovery After Photobleaching (FRAP) and Fluorescence Loss In Photobleaching (FLIP) are the two most popular fluorescence microscopy techniques. ${ }^{[23-26]}$ Interestingly, these methods are based on a photobleaching step which allows to switch the fluorescence off by high intensity illumination with a focused laser beam. However, considering the energy of the laser beam, care must be taken to avoid disruption of the cellular structure. ${ }^{[27,28]}$ On the other hand, photobleaching-based methods cannot be used to investigate events occurring at the scale of a single lipid bilayer thickness due to the poor laser beam focusing resolution $(\mu \mathrm{m})$ compared to the lipid bilayer thickness $(4 \mathrm{~nm})$. Under these conditions, discrimination between the outer and the inner leaflet of a membrane is therefore not possible. Yet, such discrimination are essential notably for the investigation of mechanisms related to the entrance and exit of active molecules across lipid membranes, a hot topic in the development of innovative pharmaceutical strategies.

In the present contribution, we focused on the development of an original combination between electrochemistry and confocal microscopy to monitor the electrochemical bleaching of fluorescently-labelled phospholipids diffusing in giant unilamellar vesicles membranes (GUVs: $10-50 \mu \mathrm{m}$ in diameter) deposited on a transparent conductive surface. For the first time, we show that electrochemistry is a powerful tool to selectively bleach the fluorescence emitted by NBD (7-nitrobenz-2-oxa-1,3-diazole) a well-known redox fluorescent probe especially used to investigate the translocation of cell-penetrating peptides. ${ }^{[29-33]}$ Importantly, compared to photobleaching techniques such as FRAP and FLIP, the electrochemical bleaching process does not affect the fluorescent probes localized "inside" the vesicles thus providing a methodology to discriminate between the outer and the inner membrane leaflets. A comparison of this original Fluorescence Loss In Electrobleaching (FLIE) procedure with FLIP established that the re-organization of the fluorescent phospholipids followed lateral diffusion dynamics, as assessed by the excellent agreement between the experimental fluorescence decay and a model established for FLIP experiments. This procedure therefore appears relevant to explore a variety of biological issues.

\section{Results and Discussion}

Development and optimization of a device allowing the preparation and the observation of asymmetrical fluorescent liposomes. The electrochemical reduction of giant unilamellar vesicles (GUVs) essentially tagged on their outer leaflets with NBD (7-nitrobenz-2-oxa-1,3-diazole), used as a model redox fluorescent probe, was first investigated. Accordingly, a first series of GUVs made of 1,2-dioleoyl-snglycero-3-phosphoglycerol (DOPG) phospholipids, a classical anionic phospholipid used to prepare endosome models, and of a small fraction (5 mol. \%) of 1,2-dipalmitoyl-sn-glycero-3phosphoethanolamine-N-(7-nitro-2-1,3-benzoxadiazol-4-yl)

(NBD-PE) was prepared. The preparation method for such asymmetrical liposomes was inspired from the works reported by Weitz, ${ }^{[34]}$ Baigl, ${ }^{[35]}$ and $\mathrm{Ces}^{[36]}$ who developed protocols to generate giant liposomes from an oil/water interface. Briefly, lipid-coated water/sucrose-in-oil (W/O) droplets (destined for the inner leaflet) were passed through an oil-water/glucose (O/W) column with an interfacial monolayer of lipids to form the vesicle outer-leaflet. The different densities between sucrose and glucose solutions allowed the W/O droplets crossing the O/W phase, forming thus giant vesicles at the bottom surface of the chamber (Figure 1; see the Supporting Information for full experimental details).

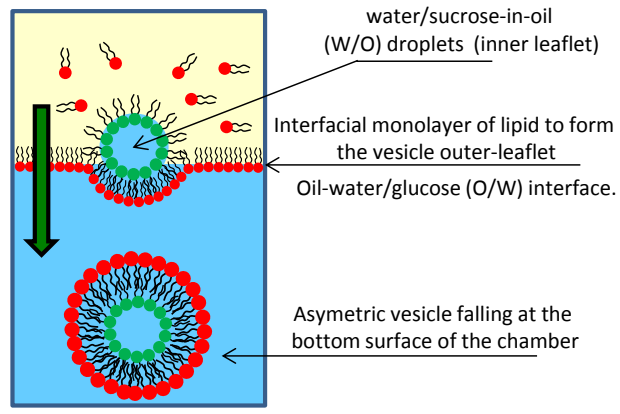

Figure 1. Schematic illustration showing the asymmetric GUV generation via phase transfer. Adapted from refs. [34, 36].

Therefore, we developed an original and specific cell made of poly(dimethylsiloxane) (PDMS) allowing not only the in situ preparation and observation of GUVs by fluorescence confocal microscopy, but also the electrochemical reduction of NBD-PE phospholipid probes (see details in S.I.). As shown in Figure 2A, a PDMS well was bonded onto a glass microscope slide previously coated with a thin layer of ITO (10 nm in thickness), a conductive and transparent electrode material commonly used in spectro-electrochemistry experiments. Nevertheless, it was observed that electrochemical properties of ITO were modified after prolonged polarizations (500 seconds) at a cathodic potential of $-0.7 \mathrm{~V} / \mathrm{Ag} / \mathrm{AgCl}$ corresponding to the value at which the NBD nitro group is reduced (vide infra). According to recent reported works, ${ }^{[37-39]}$ the cathodic polarization of ITO thin films leads to morphological, optical, and conductivity changes of the 
material due to the reduction of its metallic sites. We overcame this drawback by covering the ITO surface with a thin gold layer (20 $\mathrm{nm}$ in thickness). Chronoamperograms obtained in the presence of such ITO/Au surfaces remained much more stable than those obtained in their absence (see S.I.). Cyclic voltammetry of NBD-glycine, used as model, was performed in the same cell to determine the reduction potential value of the fluorescent probe under the same conditions as those used to bleach NBD-tagged giant vesicles. Accordingly, and in agreement with similar nitro derivatives, ${ }^{[40,41]}$ the first reduction wave of NBD was observed at $-0.7 \mathrm{~V} / \mathrm{Ag} / \mathrm{AgCl}$ (S.I.). Although light transmission of the NBD probe through the ITO/Au bilayer was only half that transmitted through an unmodified glass microscope cover slide (see S.I.), it was intense enough to visualize NBD-tagged GUVs (Figure 2B). As sketched in Figure $2 \mathrm{~A}$, a $\mathrm{Pt}$ wire and a $\mathrm{Ag} / \mathrm{AgCl}$ wire $(0.5 \mathrm{~mm}$ in diameter each) used as the counter and the reference electrodes, respectively, were inserted into the well to complete the electrochemical device. In Figure $2 \mathrm{~B}$ is represented a typical confocal microscopy image focused at the equatorial plane of a giant unilamellar vesicle composed of DOPG and of a small fraction (5 mol. \%) of NBD-PE fluorescent phospholipids on its outer leaflet. To improve the stability and integrity of vesicles (some of them were found to be opened on the gold surface i.e. having an around 10 micrometers pore size) the electrode surface was modified with a biotin monolayer (see details in S.I.). ${ }^{[42]}$ This modification affected neither the light transmission nor the electrochemical properties of the gold layer.

(A)

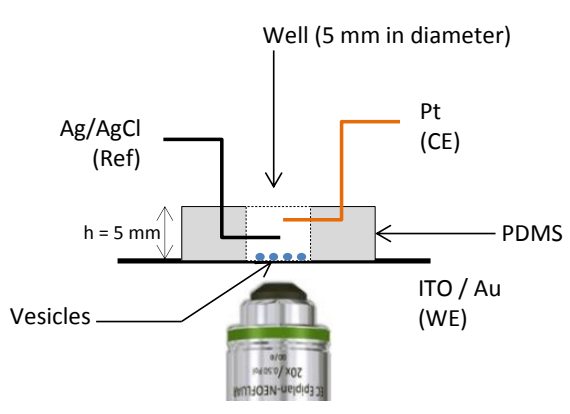

Figure 2. A) Scheme of the experimental device allowing preparation as well as visualization of giant vesicles before and during the electrochemical extinction of fluorescence. B) Typical fluorescence (top) and transmission (bottom) microscopy images focused at the equatorial plane of a giant unilamellar vesicle $(50 \mu \mathrm{m}$ in diameter) made of DOPG and of NBD-PE on its outer leaflet $(5 \mathrm{~mol} . \%)$ and settled at the bottom of the PDMS well as sketched in A).

Electrochemical bleaching of the outer leaflet of NBDlabelled giant liposomes - An original approach called Fluorescence Loss In Electrobleaching (FLIE). Our approach was validated by a first series of experiments performed in the presence of outer leaflet NBD-labelled vesicles. Typically, after verifying both the integrity of the vesicle and its contact with the electrode surface (some vesicles did not reach the bottom of the well) a confocal image was taken at the equatorial plane of the GUV. The working electrode was then polarized at a constant cathodic potential value of $-0.9 \mathrm{~V} / \mathrm{Ag} / \mathrm{AgCl}$ (i.e. $200 \mathrm{mV}$ more negative than the reduction peak of the NBD nitro group). The potentiometric pulse was interrupted after 30 seconds, and a new confocal microscopy image was taken, still focused at the equatorial plane of the vesicle (i.e., out of the electrochemicallybleached area). This sequence was repeated about 20 times on the same vesicle to follow fluorescence variations at the vesicle equatorial plane as a function of the polarization time. Figure 3 shows a typical series of confocal microscopy images obtained as a function of the reduction time and focused at the equatorial plane of a GUV containing DOPG phospholipids and a small fraction ( $5 \mathrm{~mol} \%$ ) of fluorescent phospholipids (NBD-PE) on its outer leaflet.

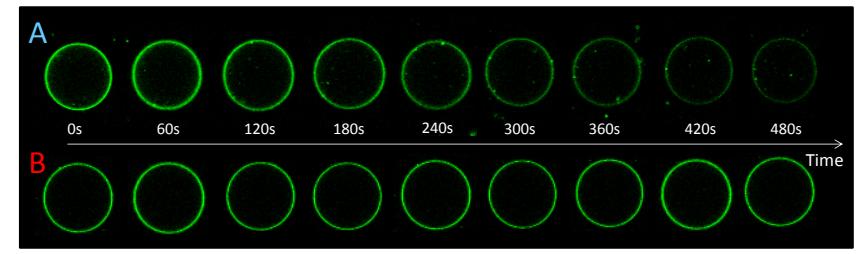

Figure 3. Typical series of confocal microscopy images focused at the equatorial plane of a GUV (50 $\mu \mathrm{m}$ in diameter) containing DOPG phospholipids and a small fraction of fluorescent phospholipids (NBD-PE; 5 mol. \%) on its outer leaflet obtained in (A) the presence and (B) the absence of cathodic polarization $(-0.9 \mathrm{~V} / \mathrm{Ag} / \mathrm{AgCl})$ of the ITO/Au surface. For simplification, only pictures recorded every minute (until 8 minutes) are shown in $(A)$ and $(B)$.

Clearly, the fluorescence intensity decreased with the number of potentiometric pulses, or in other words with the progress of the reduction reaction. Importantly, fluorescence extinction was not due to a photo-bleaching process since constant fluorescence intensities were obtained under open circuit conditions (compare $A$ and $B$ in Figure 3). This also indicates that excited NBDfluorophores were not quenched near the thin metal surface $(20$ $\mathrm{nm}$ thickness) as often observed at the surface of thick metallic materials. ${ }^{[14,15,43,44]}$ On the other hand, polarization of the working electrode at $-0.9 \mathrm{~V}$ also triggers water and oxygen reduction (see the cyclic voltammogram of NBD in the S.I.). Reduction of NBD could possibly be achieved according to a $\mathrm{H}_{2}$ mediated process, and/or $\mathrm{OH}^{-}$that is known to switch off the NBD fluorescence through a $\mathrm{pH}$ jump. ${ }^{[45,46]}$ However, the $\mathrm{pH}$ increase possibly triggered through the production of hydroxyl anions would be compensated by the generation of protons at the anode through water oxidation since the auxiliary and working electrodes are not separated in the well. Additionally, we showed that bubbling $\mathrm{H}_{2}$ in a NBD-glycine containing solution did not quench the fluorescence. Furthermore, it was impossible to quench a vesicle which was not in contact with the electrode surface, a parameter verified through $z$ stacking in confocal microscopy, demonstrating that no electrogenerated species diffusing in the bulk of the solution was able to switch NBD fluorescence off. This point also rules out the possible indirect reduction of NBD by the superoxide anion $\left(\mathrm{O}_{2}{ }^{--}\right)$or any transient 
species that would be produced in case of oxygen reduction. Moreover, at the vesicle/electrode interface, the oxygen concentration is expected to be very small when the GUV is in contact with the electrode surface. Also, it is very unlikely that superoxide anions may easily reach lipid-NBD probes located in a negatively charged (DOPG) environment. Under these conditions, the global fluorescence decrease observed at the GUV equatorial plane can be ascribed to the direct (heterogeneous) electrochemical reduction of the NBD probes in contact with the electrode surface. Note that similar fluorescence extinction decays were obtained at gold bare surfaces indicating that the electron transfer efficiency was not affected by the presence of a biotin monolayer (see S.I.).

At this stage it is noteworthy that our approach may be viewed as analogue to the Fluorescence Loss In Photobleaching (FLIP) technique in the sense that the latter consists in switching off the fluorescence by a high intensity illumination, with a focused laser beam, while fluorescence is monitored out of the bleached area. ${ }^{[23-26]}$ However, the additional interest of an electrochemical switching allows to ascertain that only molecules located in the outer leaflet can be switched (see below). By analogy with the FLIP technique, this quenching method can therefore be assimilated to a bleaching process where the laser beam is replaced by an electrode, thus prompting us to introduce the FLIE acronym (for Fluorescence Loss In Electrobleaching) to qualify this method. The initial electrochemical bleaching only occurs in the region corresponding to the surface of the GUV in contact with the electrode. Yet, the depletion caused by electrolysis generated a concentration gradient triggering a diffusion of fluorescent phospholipids towards the fluorophoredepleted region of the GUV. ${ }^{[47,48]}$ Moreover, when the polarization of the electrode was performed continuously, as in FLIP experiments, the fluorescence intensity decay overlapped the one obtained for successive polarizations (see S.I.), indicating that the fluorescence decay observed at the equatorial plane did not arise from the period of relaxation between two successive electrolyses. Indeed, this time (few seconds) is much shorter than the characteristic time of diffusion-driven relaxation (roughly hundreds of seconds; see below).

Discrimination between the outer and the inner membrane leaflets. The qualitative electrochemical bleaching of the outer leaflet of NBD-labelled giant liposomes led us then to test our approach in view of a possible discrimination between the outer and the inner membrane leaflets. Accordingly, the fluorescence extinction was then quantitatively investigated as a function of the NBD fluorescent probe localization: (a) either mainly on the outer, (b) mainly on the inner, (c) or on both inner/outer leaflets. Fluorescence variations at the equatorial plane of GUVs were corrected from background signals and photo-bleaching (see details in S.I.) and quantified as a function of the number of potentiostatic polarizations and the total time elapsed for the electrochemical reduction. Results are summarized in Figure 4, where $\mathrm{F}^{0}$ and $\mathrm{F}$ are the corrected fluorescence intensities at time 0 and at time $t$, respectively.

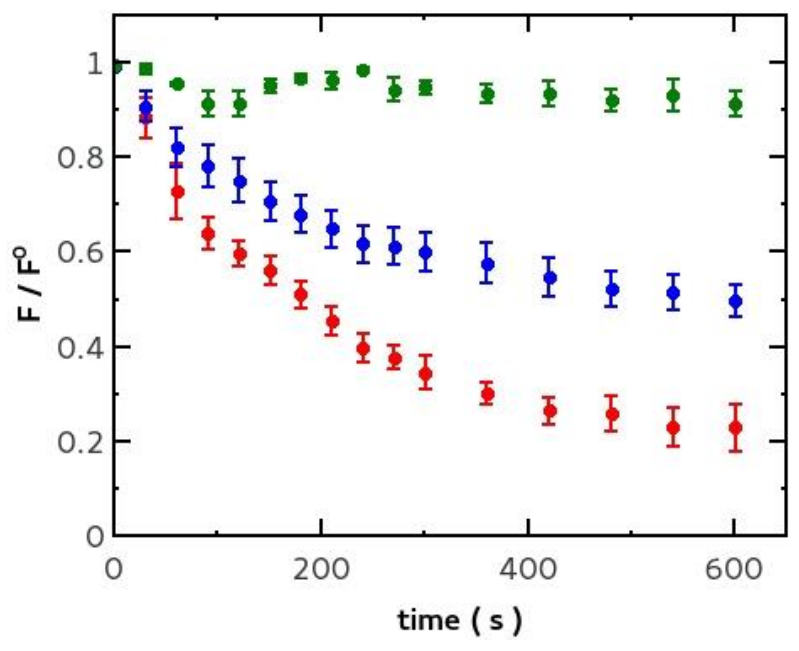

Figure 4. Fluorescence variations as a function of polarization time $(E=-0.9$ $\mathrm{V} / \mathrm{Ag} / \mathrm{AgCl})$ obtained at a biotinylated electrode surface for $(\bullet)$ inner $(\mathrm{N}=5)$, $(\bullet)$ inner/outer $(\mathrm{N}=13)$, and $(\bullet)$ outer $(\mathrm{N}=5$ ) leaflet NBD-labelled DOPG GUVs. The numbers $N$ between parentheses are those of the GUV analyzed in each case. The error bars represent the standard error (S.E.).

Three different behaviors were obtained depending on the fluorescent probe main localization. First, almost no fluorescence extinction was obtained in the presence of innerleaflet NBD-labelled vesicles demonstrating that electrochemical reduction of fluorescent probes that are inside the vesicle cannot be achieved. As already verified, the slight fluorescence loss (around $10 \%$ ) was not due to a photobleaching process. Actually, a small fraction of fluorescent phospholipids may have been incorporated in the outer leaflet of the vesicle during their preparation. ${ }^{[34,35]}$ Conversely, a significant fluorescence intensity decrease was observed in the case of outer-leaflet NBD-labelled vesicles. Almost $80 \%$ of the fluorescence was indeed quenched after 600 seconds of electrochemical reduction/bleaching. As a symmetrical situation of the preparation of inner-leaflet labelled vesicles, outer-leaflet tagged vesicles finally presented $20 \%$ of the fluorescent phospholipids inserted in the inner leaflet, a proportion consistent with reported values in ref. ${ }^{[34]}$. Accordingly, fluorescence extinction of both inner- and outer-leaflet NBDlabelled vesicles led to an average fluorescence extinction of $50 \%$. Similarly to the two previous series of experiments, some dissymmetry in the NBD-PE repartition between the two leaflets most likely happened, but they compensated. Indeed, the normalized fluorescence intensity at $t=600 \mathrm{~s}$ laid in the 0.32 0.69 range with a mean value of $0.50 \pm 0.033$ (S.E.). Note that one could wonder whether the inter-leaflet heterogeneity in terms of repartition of fluorescent phospholipids NBD-PE could arise from the dynamics of phospholipid exchange between the inner and outer leaflets. Actually, the characteristic time for lipid flip-flop is far too low to be taken into account within the time window of the electrolyses. ${ }^{[49]}$

Importantly, and contrary to the FLIP technique, these results confirmed that electrochemical bleaching only concerned fluorescent phospholipids localized on the outer-leaflet of 
vesicles allowing thus discrimination between both sides of lipid bilayers.

Fluorescent lipids re-organization upon electrochemical bleaching. The strong analogy between the FLIE assay and the FLIP technique prompted us to carry out a more thorough comparison of both techniques. It was notably important to verify whether the re-organization of the fluorescent phospholipids observed during the FLIE assay followed the expected diffusiondriven dynamics. In this context, FLIP experiments were first performed experimentally on the same GUV population and the resulting fluorescence decay simulated to determine the phospholipid lateral diffusion coefficient. In FLIP experiments presented in the following, photo-bleached zones were localized at the top of vesicles (radius a). The same results were obtained when photobleaching was performed at the bottom of vesicles i.e. where the membrane is in contact with the electrode surface. The region to be bleached was defined (disk with ableach radius) and then confocal microscopy observations were performed at the equatorial plane of GUVs allowing subsequent fluorescence loss monitoring during photo-bleaching. The bleaching consisted in sequences of 20 laser pulses ( $3 \mathrm{~s}$ duration each). The vesicle equatorial plane was imaged between successive sequences of laser pulses. A typical fluorescence decay obtained under these conditions is shown in Figure 5.

The fluorescence loss at the equator was then simulated, assuming a lateral diffusion-directed refilling of the bleached region. The model relies on the numerical solution of the diffusion equation solved on the surface of the oblate spheroidal vesicles (see S.I. for details). The diffusion coefficient value ( $D=$ $4 \mu \mathrm{m}^{2} . \mathrm{s}^{-1}$ ), obtained through the fitting procedure, was consistent with those reported for the reorganization of similar phospholipids from other methods featuring fluorescence correlation spectroscopy (FCS), ${ }^{[50]}$ single particle tracking, ${ }^{[51]}$ or FRAP[52]

The numerical fit of FLIP data presented in Figure 5, for inner/outer labelled vesicles, was then compared with FLIE data for outer-leaflet labelled vesicles since the latter technique was shown not to bleach the inside of vesicles. To be relevant the comparison of FLIP and FLIE was made with vesicles possessing similar ableach / a (bleached surface radius / vesicule radius) ratios. The value of ableach which corresponds to the membrane surface in contact with the electrode was determined from confocal images. Accordingly, these ratios were equal to 0.66 and 0.71 in FLIP and FLIE techniques, respectively.

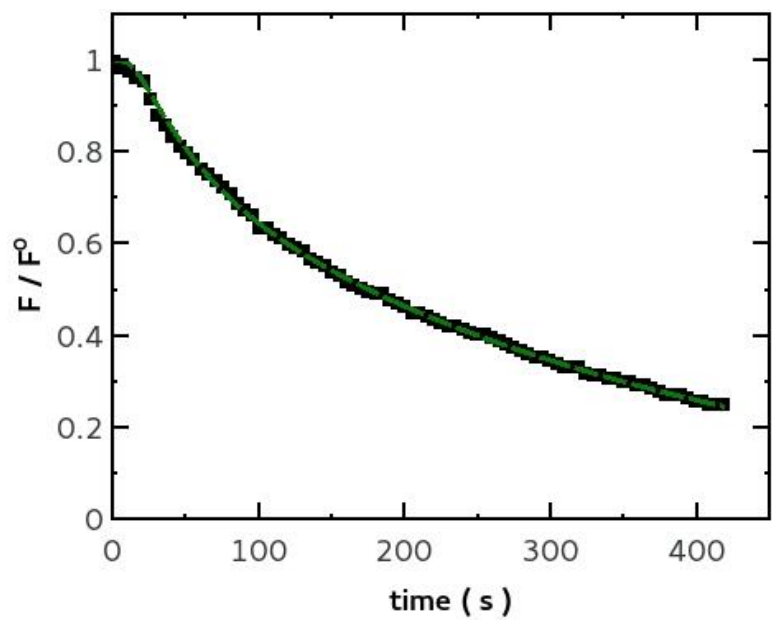

Figure 5. Fluorescence intensity decay obtained during a FLIP experiment of outer+inner leaflet NBD-labelled giant vesicle $(65 \mu \mathrm{m}$ in diameter - ableach / $\mathrm{a}=$ 0.66) - black points (experimental data) and corresponding modelization (green dashed line) of lateral diffusion driven decay of fluorescence; $D=4$ $\mu \mathrm{m}^{2} / \mathrm{s}, \mu=0.8, \alpha=0.8$, mean deviation $7 \times 10^{-4}$ per point. $\mu$ and $\alpha$ are defined in Supporting Information.

As shown in Figure 6, a good fit between experimental and simulated data was obtained confirming that the FLIE technique also generates fluorophore gradients which trigger lateral diffusion of the lipids.

Interestingly, these results show that FLIE performed on vesicles could be used to estimate diffusion coefficient values. Moreover, since FLIE only affects probes located in the outer leaflet, one can envision to study phospholipid dynamics even in asymmetrical vesicles without fast acquisition requirement as in FCS, FRAP or single particle tracking-based methods.

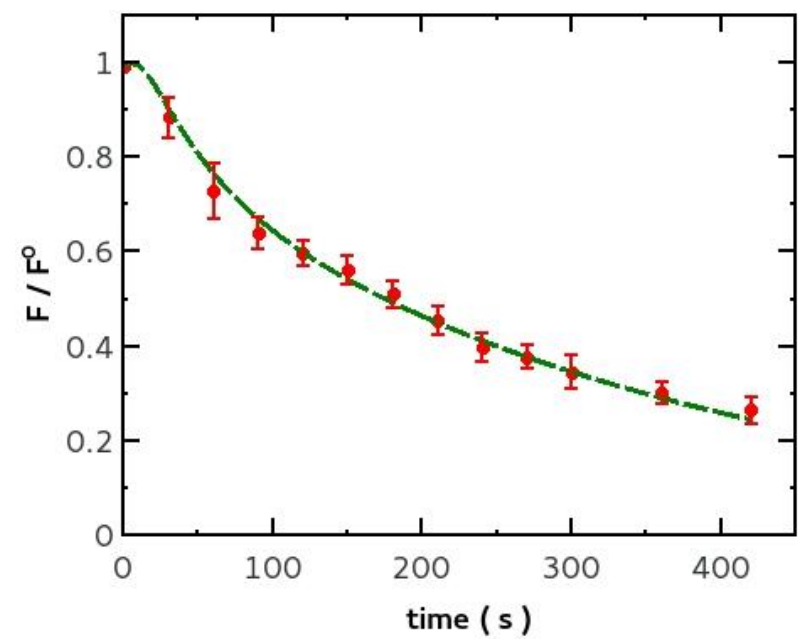

Figure 6. Comparison between the numerical model of fluorescence intensity decay obtained through the FLIP assay (green dashed line) and the fluorescence decay obtained during FLIE experiments involving outer leaflet NBD-labelled GUVs (red points) also presented in Figure 4. 


\section{Conclusions}

We have established that electrochemistry is a powerful tool to selectively bleach the fluorescence emitted by redox-active fluorophores localized on the outer leaflet of a lipid bilayer. Compared to photobleaching techniques such as FRAP and FLIP, the electrochemical bleaching process does not affect the fluorescent probes localized inside the vesicles thus providing an exploration tool allowing discrimination between the outer and the inner membrane leaflets. This electrochemical process also discards the use of chemical reducing agents that may undergo slow internalization within vesicles. ${ }^{\left[{ }^{[3]}\right]}$ Furthermore, the FLIE procedure appeared reliable to explore the re-organization dynamics of a single leaflet in real time. The capacity to totally bleach the fluorophores located in the outer leaflet also enables the possibility to monitor their passage between inner and outer leaflets (e.g. flip-flop of lipids) with confocal microscopy. This versatile approach appears very promising in the exploration of biological and pharmacological issues related to the internalization or release of analytes across lipid membranes.

\section{Experimental Section}

Lipids. 1,2-dioleoyl-sn-glycerol-3-phospho-(1'-rac-glycerol) sodium salt (DOPG, $10 \mathrm{mg} / \mathrm{mL}$ in chloroform) and 1,2-dipalmitoyl-sn-glycero-3phosphoethanolamine-N-(7-nitro-2-1,3-benzoxadiazol-4-yl) ammonium salt (NBD-PE, $1 \mathrm{mg} / \mathrm{mL}$ in chloroform, $\lambda_{\mathrm{ex}}=460 \mathrm{~nm}, \lambda_{\mathrm{em}}=535 \mathrm{~nm}$ ) were purchased from Avanti Polar Lipids.

Chemicals. Mineral oil Bio-ultra, D-(+)-Glucose, Sucrose, and Biotin were obtained from Sigma Aldrich. Phosphate buffer saline (PBS) tablets (10 mM phosphate, $150 \mathrm{mM}$ sodium chloride; $\mathrm{pH}$ 7.4) were purchased from GIBCO Invitrogen Corporation. All chemicals were used without further modification.

Solutions. Water was highly purified (resistivity $=18 \mathrm{M} \Omega . \mathrm{cm}$; Milli-Q system; Millipore, Billerica, MA, USA). Glucose and sucrose solutions were prepared by dissolving $6.3 \mathrm{~g}$ and $12 \mathrm{~g}$ of each compound, respectively, in $50 \mathrm{~mL}$ of PBS, to reach a final concentration of $0.7 \mathrm{M}$ and an osmolarity around $990 \mathrm{mOsm}$.

Device fabrication. The experimental device shown in Figure 2 is based on a home-made well inspired by the work reported by Yamada et al. ${ }^{[35}$ Details are given in the S.I.

Giant Unilamellar Vesicles (GUVs). The preparation of GUVs was based on the work reported by Yamada et al. who developed a protocol to generate liposomes from an oil/water interface. ${ }^{[35]}$ For full experimental details see the S.I.

Electrochemical experiments. Just before each experiment, and after the vesicles preparation, the electrochemical device shown in Figure 2 was completed by inserting, through the PDMS well, a $\mathrm{Ag} / \mathrm{AgCl}$ wire $(0.5$ $\mathrm{mm}$ in diameter) and a platinum wire $(0.5 \mathrm{~mm}$ in diameter) used as the reference and the counter electrode, respectively. Then, a series of electrochemical reduction were performed by applying a potential value of $-0.9 \mathrm{~V}(\mu \mathrm{AUTOLAB}$ Type III). A picture of the remaining fluorescence at the equatorial plane of the GUV was taken after every 30 seconds until $300 \mathrm{~s}$ then every 60 seconds until 600 seconds. After the end of each experiment, the vesicle integrity was verified. Details are given in the S.I. part.

Imaging of GUVs. Giant vesicles were observed by phase-contrast and confocal microscopy (Zeiss LSM 710), using EC Plan-Neofluar 10x (numerical aperture 0.3 ) and EC Plan-Neofluar 20x (numerical aperture 0.5 ) objectives. More details are given in the S.I. part.
Image analysis and fluorescence quantification. Images were analyzed with the ImageJ software (http://rsb.info.nih.gov/ij/). The average fluorescence intensity at the equatorial plane of each GUV was measured using the Azimuthal Average plugin. See S.I. for fluorescence quantification details.

FLIP experiments. FLIP experiments were performed with the same confocal fluorescence microscope as that used for GUVs imaging (Zeiss LSM 710). Photo-bleaching experiments were carried out with a circular spot using the $458 \mathrm{~nm}$ lines from a $40 \mathrm{~mW}$ argon laser operating at $100 \%$ power. In FLIP experiments, focus was made on the top of the vesicle (radius a), where it appears as a uniform disk. The region to be bleached was defined (disk with radius ableach), and focus was then made at the equatorial plane of the GUV allowing subsequent fluorescence loss monitoring during bleaching. The bleaching was performed with sequences of 20 laser pulses ( $3 \mathrm{~s}$ total duration). The vesicle equator was imaged between successive sequences of laser pulses. Fluorescence images were acquired at the GUVs equatorial plane as a function of time, at a low laser intensity $(0.2 \%$ laser power) under the following conditions: 2 or $3 x$ optical zoom, $512 \times 512$ pixel resolution, depth 8 bits, nominal speed of 9 (total scan speed $<1 \mathrm{~s}$ ). The pinhole was set at 1 Airy, corresponding to an optical section of $5 \mu \mathrm{m}$. Details on data analysis and the corresponding numerical model are given in the S.I.

\section{Acknowledgements}

This work was supported by the CNRS (UMR 8640 - PASTEUR), the French Ministry of Research, the Ecole Normale Supérieure, Université $\mathrm{P}$. et $\mathrm{M}$. Curie, and the Agence Nationale de la Recherche (grant number: ANR-12-BS08-0002-01 "ELIPTIC"). The authors thank Conacyt for support of A.I.P.J. and Prof.Dr. Christian Amatore for fruitful discussions and helpful advices.

Keywords: Electrochemical bleaching - Fluorescence $\cdot$ Lipid Vesicles $\cdot$ Confocal Microscopy $\cdot$ Diffusion of phospholipids.

[1] P. Audebert, F. Miomandre, Chem. Sci. 2013, 4, 575-584

[2] R.C. Engstrom, S. Ghaffari, H. Qu, Anal. Chem. 1992, 64, 2525-2529.

[3] W.J. Bowyer, J. Xie, R.C. Engstrom, Anal. Chem. 1996, 68, 2005-2009.

[4] J.E. Vitt, R.C. Engstrom, Anal. Chem. 1997, 69, 1070-1076.

[5] C. Amatore, F. Bonhomme, J.L. Bruneel, L. Servant, L. Thouin, Electrochem. Commun. 2000, 2, 235-239.

[6] C. Amatore, F. Bonhomme, J.L. Bruneel, L. Servant, L. Thouin, J. Electroanal. Chem. 2000, 484, 1-17.

[7] S. Cannan, I.D. Macklam, P.R. Unwin, Electrochem. Commun. 2002, 4, 886-892.

[8] F.-M. Boldt, J. Heinze, M. Diez, J. Petersen, M. Börsch, Anal. Chem. 2004, 76, 3473-3481.

[9] C. Amatore, A. Chovin, P. Garrigue, L. Servant, N. Sojic, S. Szunerits, L. Thouin, Anal. Chem. 2004, 76, 7202-7210.

[10] T. Doneux, L. Bouffier, B. Goudeau, S. Arbault, Anal. Chem. 2016, 88, 6292-6300

[11] J.P. Guerrette, S.J. Percival, B. Zhang, J. Am. Chem. Soc. 2013, 135, 855-861.

[12] S.M. Oja, J.P. Guerrette, M.R. David, B. Zhang, Anal. Chem. 2014, 86 6040-6048.

[13] S.M. Oja, B. Zhang, Anal. Chem. 2014, 86, 12299-12307.

[14] J.R. Casanova-Moreno, D. Bizzotto, Langmuir 2013, 29, 2065-2074

[15] Z.L. Yu, J.R. Casanova-Moreno, I. Guryanov, F. Maran, D. Bizotto, J. Am. Chem. Soc. 2015, 137, 276-288.

[16] A. Musgrove, D. Bizzotto, Langmuir 2015, 31, 12797-12806. 
[17] R.R.C. New, "liposomes: A Practical Approach"; Oxford University Press: Oxford, England, 1990.

[18] E. Sackmann, in "Structure and Dynamics of Membranes: From Cells to Vesicles"; Lipowsky, R., Sackmann, E.; Eds.; Elsevier Science B.V.: Amsterdam, The Netherlands, 1995; Vol. 1, pp 1-63.

[19] P.L. Luisi, P. Walde, "Giant Vesicles"; John Wiley \& Sons Ltd. : New York, 2000.

[20] P. Messina, F. Lemaître, F. Huet, K.A. Ngo, V. Vivier, E. Labbé, O. Buriez, C. Amatore, Angew. Chem. Int. Ed. 2014, 53, 3192 -3196.

[21] W. Zhan, A.J. Bard. Anal. Chem. 2006, 78, 726-733.

[22] J.B. Pawley, Handbook of Biological Confocal Microscopy, 2006, 3rd Edition, Springer Science+Business Media, LLC, New York, USA.

[23] D. Axelrod, D.E. Koppel, J. Schlessinger, E. Elson, W.W. Webb, Biophys. J. 1976, 16, 1055-1069.

[24] D.E. Koppel, D. Axelrod, J. Schlessinger, E. Elson, W.W. Webb, Biophys. J. 1976, 16, 1315-1329.

[25] J. Lippincott-Schwartz, E. Snapp, A. Kenworthy, Nat. Rev. Mol. Cell. Biol. 2001, 2, 444-456.

[26] D. Chen, S. Huang, J. Cell. Biol. 2001, 153, 169-176

[27] J.A. Bloom, W.W. Webb, J. Histochem. Cytochem. 1984, 32, 608-616.

[28] A. Flock, B. Flock, E. Scarfone, J. Neurocytol. 1998, 27,507-516.

[29] S.B. Fonseca, M.P. Pereira, S.O. Kelley, Adv. Drug. Deliv. Rev. 2009, 61, 953-964

[30] S. El-Andaloussi, T. Holm, Ü. Langel, Curr. Pharm. Des. 2005, 11 3597-3611.

[31] C. Bechara, S. Sagan, FEBS Lett. 2013, 587, 1693-1702.

[32] J.-M. Swiecicki, M. Di Pisa, F. Lippi, S. Chwetzoff, C. Mansuy, G. Trugnan, G. Chassaing, S. Lavielle, F. Burlina, Chem. Commun. 2015 51, 14656-14659.

[33] J.-M. Swiecicki, A. Bartsch, J. Tailhades, M. Di Pisa, B. Heller, G. Chassaing, C. Mansuy, F. Burlina, S. Lavielle, ChemBioChem. 2014 15, 884-891.

[34] S. Pautot, B.J. Frisken, D.A. Weitz, Proc. Natl. Acad. Sci. USA. 2003 100, 10718-10721.

[35] A. Yamada, M. Le Berre, K. Yoshikawa, D. Baigl, ChemBioChem. 2007 8, 2215-2218

[36] Y. Elani, S. Purushothaman, P.J. Booth, J.M. Seddon, N.J. Brooks, R.V. Lawa, O. Ces, Chem. Commun. 2015, 51, 6976-6979.
[37] M. Senthilkumar, J. Mathiyarasu, J. Joseph, K.L.N. Phani, V. Yegnaraman, Mater. Chem. Phys. 2008, 108, 403-407.

[38] E. Matveeva, J.Electrochem. Soc. 2005, 152, H138-H145.

[39] C.C. Pla Cid, E.R. Spada, M.L. Sartorelli, Appl. Surf. Sci. 2013, 273 603- 606.

[40] S. Bollo, P. Jara-Ulloa, G. Zapata-Torres, E. Cutino, J.C. Sturn, L.J. Nuñez-Vergara, J.A. Squella, Electrochim. Acta. 2010, 55, 4558-4566.

[41] J.A. Squella, S. Bollo, L.J. Nuñez-Vergara, Curr. Org. Chem. 2005, 9 , 565-581.

[42] H.M. Laborde, A.M.N. Lima, F.C.C.L. Loureiro, C. Thirstrup, H. Neff Thin Solid Films, 2013, 540, 221-226.

[43] R.R. Chance, A.H. Miller, A. Prock, R. Silbey, J. Chem. Phys. 1975, 63, 1589-1595

[44] J.R. Lakowicz, Anal. Biochem. 2005, 337, 171-194.

[45] J.-M. Swiecicki, M. Di Pisa, F. Burlina, P. Lécorché, C. Mansuy, G. Chassaing, S. Lavielle, Biopolymers 2015, 104, 533-543.

[46] R. de Oliveira, M. Durand, L. Challier, P. Messina, J.-M. Swiecicki, M. Di Pisa, G. Chassaing, S. Lavielle, O. Buriez, E. Labbé, J. Electroanal. Chem. Under revision.

[47] C. Amatore, Y. Bouret, E. Maisonhaute, J.I. Goldsmith, H.D. Abruna Chem. Eur. J. 2001, 7,2206-2226.

[48] C. Amatore, Y. Bouret, E. Maisonhaute, J.I. Goldsmith, H.D. Abruna, ChemPhysChem. 2001, 2, 130-134.

[49] F.X. Contreras, L. Sánchez-Magraner, A. Alonso, F.M. Goñi, FEBS Letters, 2010, 584, 1779-1786

[50] K. Bacia, C.G. Schuette, N. Kahya, R. Jahn, P. Schwille, J Biol Chem. 2004, 279, 37951-37955.

[51] Y.A. Domanov, S. Aimon, G.E.S. Toombes, M. Renner, F. Quemeneur A. Triller, M.S. Turner, P. Bassereau, Proc. Natl. Acad. Sci. U.S.A 2011, 108, 12605-12610.

[52] F. Pincet, V. Adrien, R. Yang, J. Delacotte, J.E. Rothman, W. Urbach D. Tareste, PLoS One 2016, 11, e0158457.

[53] M.J. Moreno, L.M.B.B. Estronca, W.L.C. Vaz, Biophys. J. 2006, 91 873-881. 